\title{
Mapping atomic electric fields and charge densities by four-dimensional STEM
}

Knut Müller-Caspary $^{1}$, M. Duchamp ${ }^{2}$, F.F. Krause ${ }^{3}$, A. Beche ${ }^{1}$, F. Winkler ${ }^{2}$, S. Löffler ${ }^{4}$, H. Soltau ${ }^{5}$, J. Zweck $^{6}$, P. Schattschneider ${ }^{7}$, J. $^{2}$ Verbeeck $^{1}$, S. van Aert ${ }^{1}$, R.E. Dunin-Borkowski ${ }^{2}$, A. Rosenauer ${ }^{3}$

${ }^{1}$ EMAT, Universiteit Antwerpen, Antwerpen, Belgium, ${ }^{2}$ Forschungszentrum Jülich, Jülich, Germany, ${ }^{3}$ IFP, University of Bremen, Bremen, Germany, ${ }^{4}$ USTEM, TU Wien, Wien, Austria, ${ }^{5}$ PN Detector GmbH, Munich, Germany, ${ }^{6}$ University of Regensburg, Regensburg, Germany, 7IFP, TU Wien, Wien, Austria

E-mail: Knut.Muller-Caspary@uantwerpen.be

We show in theory, simulation and experiment how atomic electric fields and charge densities can be measured by 4D-STEM. In this imaging mode, 2D diffraction patterns are recorded on a pixelated detector at a 2D STEM raster. In quantum mechanics, the first moment $p$ of a diffraction pattern is related to the expectation value for the momentum transfer and provides a quantitative measure of the angular deflection of the STEM probe in electric or magnetic fields [1]. This overcomes ambiguities in conventional differential phase contrast STEM where segmented detectors record portions of the diffraction pattern and average over large angular domains [2].

Our concept is explained in figure (a), schematically showing a focused STEM probe positioned at (1) a nearly field-free region and (2) close to an atom where the projected electric field $\mathrm{Ep}$ is nonzero. Whereas the propagation of the wave in case (1) is preserved as illustrated by the wave fronts within the STEM illumination cone, the interaction with the electric field Ep for situation (2) causes both a distorted wave front and a deflection to the right. Assuming a Ga column in a GaN crystal with $1.3 \mathrm{~nm}$ thickness, we simulated the diffraction patterns (Ronchigrams) on the right and determined the first moments $p$ as indicated. This demonstrates how the complexity of the Ronchigram condenses to a single vector with fundamental physical meaning.

Due to Ehrenfest's theorem, $\mathrm{p}$ is proportional to the expectation value of the electric field. For sufficiently thin specimens, we found that $\mathrm{p}$ is also proportional to the projection of the electric field Ep, convolved with the incident probe intensity. Furthermore, its divergence directly yields the projected charge density, convolved with the probe intensity [1,3].

An early 4D STEM experiment for Strontium Titanate is shown in figure (b), where a slow-scan CCD camera was employed to raster a unit cell with $20 \times 20$ pixels. The redistributing Ronchigram intensity in the vicinity of the atomic columns is clearly seen on the left. Determining the first moments and subsequently the electric field, we obtain the atomically resolved electric field map on the right. As expected from the screened nuclear charge, atoms appear as sources of the electric field, their magnitude being determined by the atomic number.

We then present recent 4D-STEM results on 2D sheets of MoS2 employing an ultrafast camera with $4 \mathrm{kHz}$ frame rate. Figure (c) depicts the (projected) charge density measured at a mono-/bilayer (ML/BL) edge with unit cell averages for both the $\mathrm{ML}$ and $\mathrm{BL}$ region. By comparison with DFT and 4D STEM simulations we show that the data agrees with theory quantitatively. In particular, we find a $2 \mathrm{R}$-like stacking of the $\mathrm{BL}$ and a Mo-terminated $\mathrm{ML} / \mathrm{BL}$ edge, which is discussed as to potential optical and catalytic properties.

With the ability to map atomic electric fields and charge densities directly without structural input, aberration-corrected 4DSTEM can shed light on the electrical configuration of vacancies, dopant atoms, bonding or polarisation fields in piezoelectric materials in future, which we exemplarily discuss using simulations [3].

[0] Martin Huth, Sebastian Ihle, Robert Ritz (PN Detector $\mathrm{GmbH}$ ) contributed to recording the data.

[1] Müller, K. et al. (2014). Nature Communications, 5, 5653:1-8. [2] Shibata, N. et al. (2012). Nature Physics, 8, 611-615.

[3] Müller, K. \& Krause, F.F. et al. (2016). Ultramicroscopy, 10.1016/j.ultramic.2016.05.004.

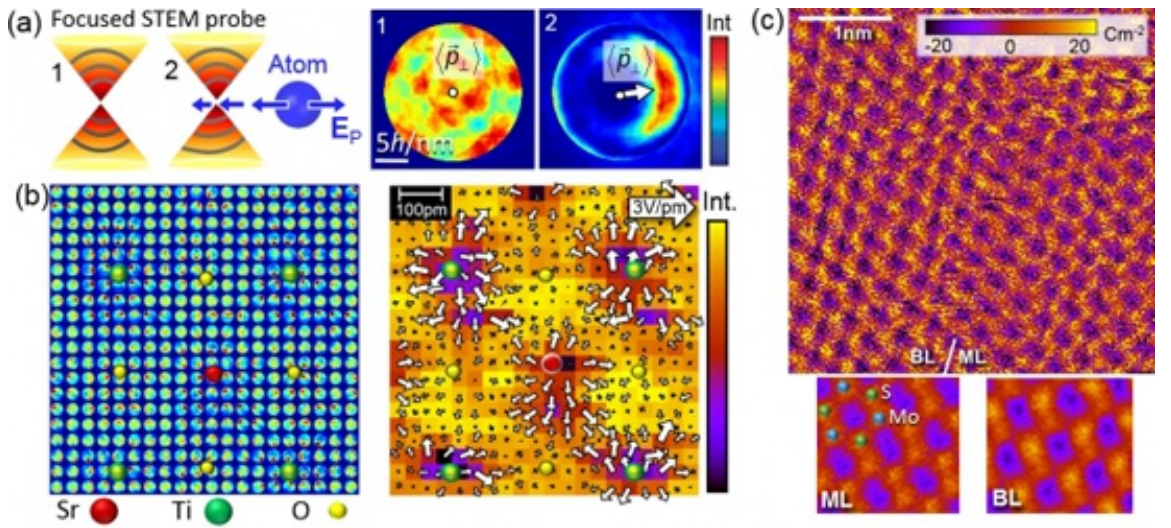

Figures. (a) Left: Illustration of a focused probe propagating through (1) a field-free region, (2) a region with nonzero electric field $E_{p}$. Right: Corresponding Ronchigrams (GaN simulation), with first moment vectors $\langle p\rangle$. $h$ : Planck constant. (b) Experimental proof of the method using STO. Left: Ronchigrams, showing the redistribution of intensity. Right: Electric field on top of the bright field STEM signal. (c) Top: Charge density across an $\mathrm{MoS}_{2} \mathrm{ML} / \mathrm{BL}$ edge. Bottom: Unit cell averages for $\mathrm{ML} / \mathrm{BL}$ regions separately including atom types.

Keywords: STEM, DPC, electric fields

Acta Cryst. (2017). A73, C119 